\title{
The burden of musculoskeletal diseases in the general population of Spain: results from a national survey
}

\author{
L Carmona, J Ballina, R Gabriel, A Laffon, on behalf of the EPISER Study Group*
}

\begin{abstract}
Objective-The objective of the EPISER study was to estimate the prevalence of rheumatoid arthritis (RA), low back pain, hand and knee osteoarthritis (OA), and fibromyalgia in the adult Spanish population, and to assess the impact of these diseases on function and quality of life, and use of health and social resources.

Methods-2998 subjects aged 20 years or above were randomly selected by stratified multistage cluster sampling from the censuses of 20 municipalities. Trained rheumatologists carried out structured visits at which subjects were asked about rheumatic symptoms and sociodemographic characteristics, completed validated instruments for measuring function (HAQ) and quality of life (SF-12), and underwent a standardised physical examination. Cases were defined by previously validated criteria.
\end{abstract}

Results-The estimated prevalences with 95\% confidence intervals were as follows: RA lifetime cumulative: $0.5 \%(0.3$ to 0.9$)$; low back pain: $14.8 \%$ (12.2 to 17.4$)$; symptomatic knee OA: $10.2 \%$ (8.5 to 11.9$)$; hand OA: $6.2 \%$ (5.9 to 6.5 ); fibromyalgia: $2.4 \%$ (1.5 to 3.2). Most conditions significantly impaired function and quality of life.

Conclusions-The EPISER study has internal and external validity for application of the results to the adult Spanish population. The diseases studied affect a significant proportion of the population, with various degrees of impact on disability and quality of life resulting in a significant number of physician visits, work disability, and medication use.

(Ann Rheum Dis 2001;60:1040-1045)

Hospital Universitario

de La Princesa,

Madrid, Spain

R Gabriel

Department of

Rheumatology,

Hospital Universitario

de La Princesa,

Madrid, Spain

A Laffon

Correspondence to: Dr L Carmona, Sociedad

Española de Reumatología,

Calle Recoletos 9, 1A, 28001

Madrid, Spain

lcarmona@ser.es

Accepted 4 April 2001

${ }^{\star}$ Members of the EPISER Study Group are shown in the appendix.
Most of the epidemiological data on rheumatic diseases at the national level come either from a small number of sampling areas considered representative of the country or are secondary data from national health surveys in which information about rheumatic diseases relies mainly on self-reporting. ${ }^{1-8}$

In Spain epidemiological studies on rheumatic diseases are scarce, despite their recognised socioeconomic impact. ${ }^{9}$ There are no systematic community or hospital registries of rheumatic diseases, and the feeling is that general knowledge about musculoskeletal diseases is low, even among health professionals. No study has ever been conducted to assess quality of life and impact on function at the population level in Spain. In fact, comparative data on the impact on quality of life measures of rheumatic diseases and other chronic conditions in population based studies are practically non-existent in most countries, despite the fact that quality of life is the most important indicator of population impact of this group of diseases. ${ }^{10}$

The Spanish Society of Rheumatology foresaw the need to assess the impact of rheumatic diseases on the general population in terms of prevalence, effect on quality of life and function, and use of health resources. The EPISER study was conducted to estimate the prevalence of specific rheumatic diseases-low back pain, rheumatoid arthritis (RA), knee and hand osteoarthritis (OA), and fibromyalgia (FM) - which were considered a priority because of the lack of reliable estimates in Spain, and to measure their social and health related impact.

\section{Subjects and methods}

SAMPLING

To obtain a probabilistic sample from which to infer national estimates, stratified cluster sampling was used. The 19 autonomic communities into which Spain is divided were assembled into eight strata with a known population. Each stratum consisted of at least four Spanish provinces with geographical and historical boundaries. The Canary Islands, which are located too far from any peninsular region, were included in the less inhabited stratum. Two or three provinces were randomly selected within each stratum and one city within each province, up to a total of 20 cities or villages. Figure 1 shows the places from which the sample was drawn. The selection process observed the rural/urban ratio of 25:75 present in Spain, and cities were defined a towns with over 10000 inhabitants. The study sample was finally extracted at random from city registers using sex and age groups which were proportional to those of the 


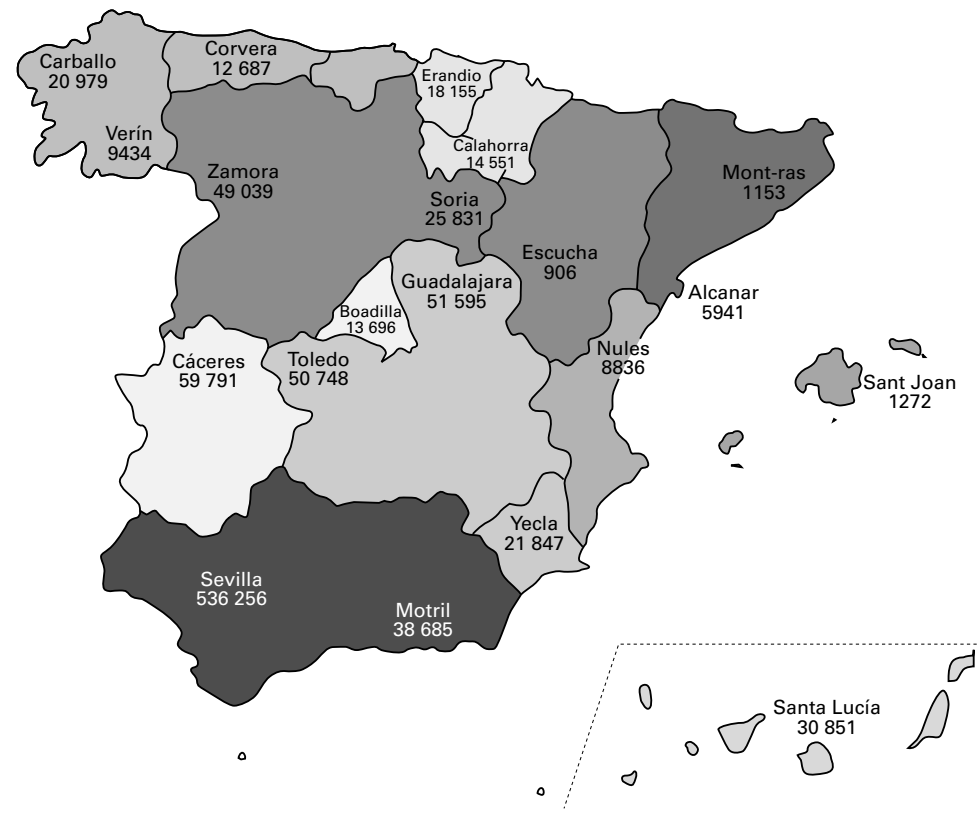

Figure 1 Map of Spain showing the 20 municipalities selected for the EPISER study. Each area in the same shade represents one stratum. Under the name of the city or village selected, the number of the eligible population ( $>20$ years) is shown.

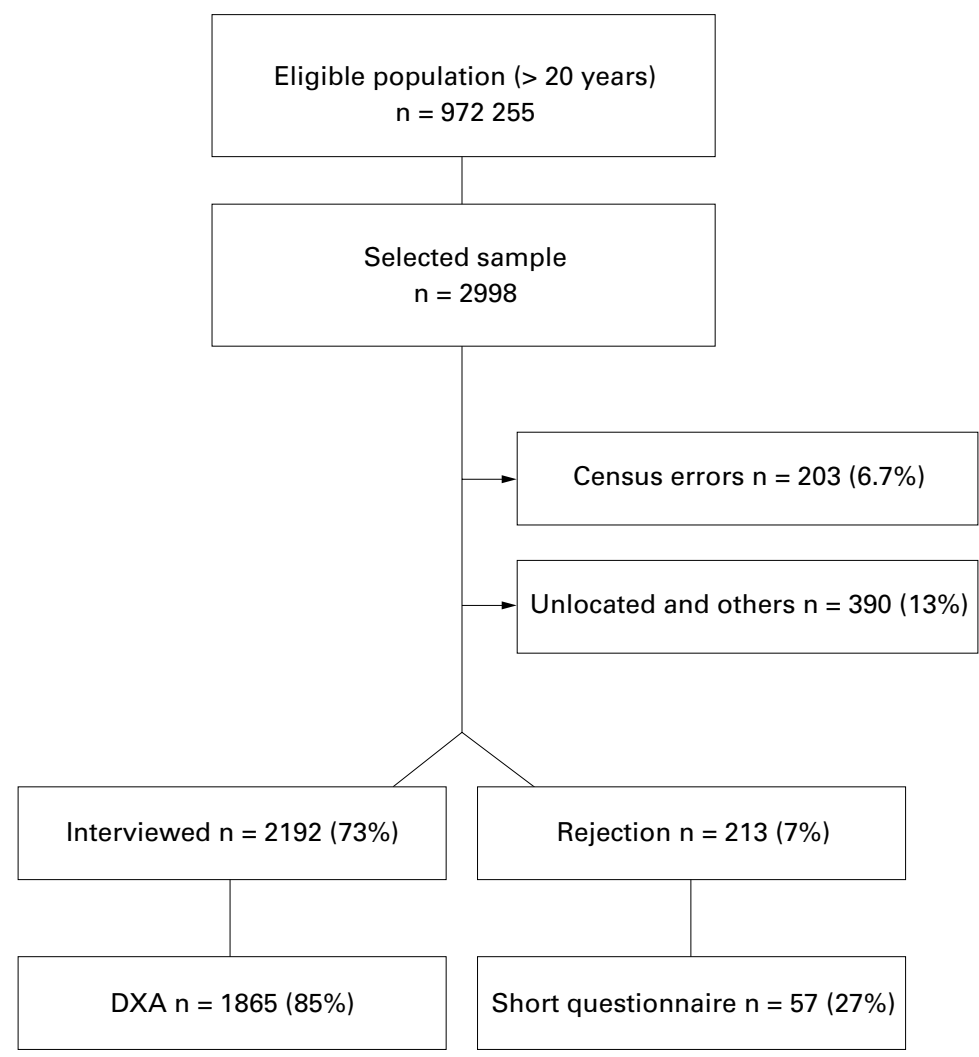

Figure 2 EPISER study: flow chart of response to the survey.

total Spanish population. Sampling was done without replacement.

RECRUITMENT

All selected subjects were informed about the study by letter. News of the study appeared in local newspapers, television, and radio shortly after the letter was sent out in order to enhance the response. Subjects then received a second letter giving them an appointment with a rheumatologist, and telephone contact was made with all subjects to confirm the appointment and to facilitate recruitment. Twenty rheumatologists were instructed by a social psychologist in recruitment strategies and were responsible for the complete implementation of the study at the local level. They were also instructed in how to conduct the interviews and subsequent examinations following a standard protocol.

A total of 2998 letters were sent to the randomly selected subjects at the addresses registered on the city register. Figure 2 shows a flow chart with the response rate obtained. The percentage of confirmed census errors (dead, moved, younger than 20) and of those who did not respond and could not be reached by other means (probable census errors) were evenly distributed between the various sex and age groups. The response was higher in villages $(83.7 \%)$, where people could be easily located by means other than mail or telephone, than in cities $(69.7 \%)$. After successful contact by telephone 213 persons refused to participate in the study (rejection rate $7 \%$ ). A short questionnaire with questions on basic sociodemographic characteristics and previous diagnosis of rheumatic diseases by a physician was obtained from 57 (27\%) of those who declined to participate. A few short questionnaires $(n=22)$ were completed by telephone at the time of refusal and the rest after mailing the questionnaire to a random sample of 100 nonresponders. After testing the distribution of previous diagnoses and sociodemographic questions, the only significant difference between participants and those who refused to participate was the place of residence, with more refusing to participate from cities than from villages.

A total of 2192 interviews were completed. In $1.6 \%$ the interviews were addressed to the main caregiver of the person selected, mainly owing to communication problems. Questions dealing with subjective issues were omitted in these cases.

Table 1 shows the distribution of the studied sample and a comparison of the data with those for the Spanish population obtained from the Institute of National Statistics. ${ }^{11}$

\section{MEASUREMENTS}

The interviews were performed at local primary care facilities after permission was obtained from the local authorities. Subjects were asked about their current health status, whether they had been given a diagnosis of any chronic disease, and whether they were receiving any treatment. All completed the Spanish validated versions of the Short Form $12(\mathrm{SF}-12)^{13}$ and Health Assessment Questionnaire (HAQ) ${ }^{14}$ to assess health related quality of life and functional capacity, respectively. Various sections of the interview asked about rheumatic symptoms. An adaptation of the screening questionnaire for RA developed by MacGregor et $a l^{15}$ was also included. This screening instrument was originally validated in a British population. On the judgment of the investigators, the adaptation 
Table 1 Comparative data of the sample in the EPISER study and of the Spanish general population

\begin{tabular}{|c|c|c|}
\hline Variable & Frequency & Population * \\
\hline Women & $1178(53.7 \%)$ & $51.12 \%$ \\
\hline \multicolumn{3}{|l|}{ Age group $(>20)$} \\
\hline $20-29$ & $463(21.1 \%)$ & $20.8 \%$ \\
\hline $30-39$ & $439(20.0 \%)$ & $20.3 \%$ \\
\hline $40-49$ & $371(16.9 \%)$ & $17.0 \%$ \\
\hline $50-59$ & $326(14.9 \%)$ & $14.3 \%$ \\
\hline $60-69$ & $313(14.3 \%)$ & $12.7 \%$ \\
\hline $70-79$ & $205(9.4 \%)$ & $10.0 \%$ \\
\hline$\geqslant 80$ & $75(3.4 \%)$ & $4.8 \%$ \\
\hline Living in cities & $1584(72.3 \%)$ & $74.0 \%$ \\
\hline \multicolumn{3}{|l|}{ Study level } \\
\hline No formal education & $271(12.4 \%)$ & $14.8 \%$ \\
\hline Elementary & $953(43.7 \%)$ & $45.9 \%$ \\
\hline High school & $398(18.2 \%)$ & $15.5 \%$ \\
\hline College & $560(25.6 \%)$ & $20.9 \%$ \\
\hline \multicolumn{3}{|l|}{ Type of job } \\
\hline Agriculture & $193(12.0 \%)$ & $8.4 \%$ \\
\hline Industry & $344(21.4 \%)$ & $20.2 \%$ \\
\hline Construction & $107(6.7 \%)$ & $9.7 \%$ \\
\hline Services & $964(60.0 \%)$ & $61.7 \%$ \\
\hline \multicolumn{3}{|l|}{ Social class $\dagger$} \\
\hline Low & $588(26.8 \%)$ & No comparable data \\
\hline Medium & $778(35.5 \%)$ & \\
\hline High & $237(10.8 \%)$ & \\
\hline Unspecified & $589(26.9 \%)$ & \\
\hline \multicolumn{3}{|l|}{ Employment status } \\
\hline Employed & $1132(51.6 \%)$ & $41.8 \%$ \\
\hline Unemployed & $74(3.4 \%)$ & $9.0 \% \ddagger$ \\
\hline Student & $107(4.9 \%)$ & $5.1 \% \S$ \\
\hline Retired & $483(22.0 \%)$ & Undetermined \\
\hline Housewives & $60(2.7 \%)$ & Undetermined \\
\hline Disabled & $107(4.9 \%)$ & $(4.6 \%)$ \\
\hline Others or unspecified & $15(0.7 \%)$ & Undetermined \\
\hline
\end{tabular}

*Data from the Institute of National Statistics, 1999 or 1997 , depending on last data available. ${ }^{11}$

†Social class was assigned depending on the profession of the respondent according to the protocol of the Spanish Society of Epidemiology. ${ }^{12}$

$\ddagger$ Much of the difference could be explained by the fact that many of the people studying in Spain sign up at the employment offices as "unemployed", while in the EPISER study students were not permitted to define themselves as "unemployed". Obtained by dividing the total number of students registered at universities and professional colleges by the number of persons over 20 .

into Spanish was very straightforward for such a short questionnaire with no loss of meaning, so no further validation was needed. Subjects were questioned about the intake of a list of antirheumatic drugs including non-steroidal antiinflammatory drugs (NSAIDs) for more than a month in total, and about the need to visit a physician because of musculoskeletal symptoms in the preceding year. All subjects suspected of having RA from their answers to the previous questions underwent a standardised physical examination to confirm the classification criteria and, if there was still a firm suspicion but not a sufficient number of classification criteria, hand $x$ rays and a latex agglutination test were performed to determine rheumatoid factor positivity. The complete interview was pilot tested in 15 patients and their accompanying relatives in a Rheumatology Clinic (Hospital de

Table 2 Distribution of the prevalence of selected rheumatic diseases in the Spanish population aged 20 and over by sex

\begin{tabular}{lcccc}
\hline & & \multicolumn{2}{l}{ Total $^{\star}$} \\
\cline { 4 - 5 } & Women & Men & Prevalence & $95 \%$ CI \\
\hline Rheumatoid arthritis & $9(0.8)$ & $2(0.2)$ & $11(0.5)$ & 0.3 to 0.9 \\
Low back pain & $210(17.8)$ & $115(11.3)$ & $325(14.8)$ & 12.2 to 17.4 \\
Knee osteoarthritis & $165(14.0)$ & $58(5.7)$ & $223(10.2)$ & 8.5 to 11.9 \\
Hand osteoarthritis & $112(9.5)$ & $24(2.3)$ & $136(6.2)$ & 5.9 to 6.5 \\
Fibromyalgia & $50(4.2)$ & $2(0.2)$ & $52(2.4)$ & 1.5 to 3.2 \\
\hline
\end{tabular}

^Estimates and confidence intervals are weighted (see Methods). la Princesa) and volunteers of varying educational backgrounds to assess the mean duration and comprehensibility.

\section{CASE DEFINITION}

Low back pain was defined by self-report. The interviewers were instructed to indicate what was understood by low back and then to ask about pain in that area. Symptomatic knee and hand OA, as well as FM, were defined by the respective ACR criteria. ${ }^{16-18} \mathrm{RA}$ cases were defined by the ACR classification criteria adapted to epidemiological surveys by MacGregor et al. ${ }^{19}$ This definition captures cases that were both active and inactive at the time of the survey.

ANALYSIS OF DATA

The sample size was chosen to achieve a $95 \%$ confidence interval in the estimate of the prevalence of RA if the prevalence was $0.5-1 \%$, taking into account an expected 30\% dropout rate and $20 \%$ to account for the effect of design. For the calculation of the confidence intervals, subjects were assigned a weight derived from the sampling scheme and the size of the eligible population of her or his place of residence. Prevalence estimates and confidence intervals were weighted and were obtained with CSAMPle (Epi Info 6.04b, Centers for Disease Control \& Prevention (CDC), USA, 1997).

The effect of specific conditions on quality of life and function was assessed by multifactorial ANOVA, controlling for covariates which previously had been found to be associated with the scores of the respective questionnaires. Specific conditions were introduced in the models as fixed factors. To test the bivariate association of factors with the HAQ score, non-parametric tests were used. The bivariate analysis of SF-12 used parametric tests. The results are reported as mean (SD) values unless otherwise indicated.

\section{QUALITY CONTROL}

All interviewers had to attend a standardisation course in which they were instructed how to conduct the interview and the examinations per protocol. In situ monitor visits were performed during the data collection. Data collection forms were further monitored centrally to check for missing data or inconsistencies. The effect of the interviewer on the results was tested in a logistic model in which age, sex, and rural/ urban setting were added as covariates.

\section{ETHICAL ISSUES}

The study was reviewed by the local ethical committee and by the evaluators of the Research Fund of the Social Security. The data set used for the random sampling and location of participants, which was discarded once the subjects were contacted, had been registered at the Official Agency for Data Protection, according to Spanish regulations.

\section{Results}

There were no significant differences in the sociodemographic factors between the study subjects and the population of Spain (table 1), 
Table 3 Distribution of the frequency of selected rheumatic diseases in the Spanish population by age

\begin{tabular}{lccccccc}
\hline \multicolumn{7}{l}{ Age bands } \\
\cline { 2 - 7 } Disease & $20-29$ & $30-39$ & $40-49$ & $50-59$ & $60-69$ & $70-79$ & $\geqslant 80$ \\
\hline Rheumatoid arthritis & $1(0.2)$ & $1(0.2)$ & $2(0.5)$ & $1(0.3)$ & $3(1.0)$ & $1(0.5)$ & $1(2.7)$ \\
Low back pain & $29(8.9)$ & $53(16.3)$ & $57(17.5)$ & $64(19.7)$ & $69(21.2)$ & $40(12.3)$ & $13(4.0)$ \\
Knee osteoarthritis & $2(0.4)$ & $3(0.7)$ & $13(3.5)$ & $32(9.8)$ & $88(28.1)$ & $69(33.7)$ & $16(21.3)$ \\
Hand osteoarthritis & - & $-1(1.1)$ & $22(6.7)$ & $48(15.3)$ & $49(23.9)$ & $13(17.3)$ \\
Fibromyalgia & - & $7(1.6)$ & $18(4.9)$ & $12(3.7)$ & $9(2.9)$ & $6(2.9)$ & - \\
\hline
\end{tabular}

and the nature of the sampling, which was probabilistic at all stages, ensured that the inferences made corresponded to national estimates.

The results showed that, by self-report, $22.6 \%$ (95\% CI 19.5 to 25.7 ) of the population in Spain aged $>20$ had a diagnosis of a chronic rheumatic disease. Of note, 171 of the cases detected did not have a previous diagnosis. On the other hand, 256 subjects who reported a chronic rheumatic disease were not identified as cases for any of the target diseases $(50.6 \%$ of all persons with a self-report of chronic rheumatic disease).

The prevalence rates of the different conditions are shown according to sex and age in tables 2 and 3, respectively. In order of frequency, the estimated prevalence of the studied diseases are: low back pain 14.8\% (95\% CI 12.2 to 17.4 ); symptomatic knee OA $10.2 \%$ (95\% CI 8.5 to 11.9$)$; symptomatic hand OA $6.2 \%$ (95\% CI 5.9 to 6.5 ); fibromyalgia $2.4 \%$ (95\% CI 1.5 to 3.2$)$; and RA $0.5 \%$ (95\% CI 0.3 to 0.9 ). All musculoskeletal conditions were significantly more frequent in women than in men $(\mathrm{p}<0.01)$ and increased

Table 4 Association of specific musculoskeletal diseases with the score obtained in questionnaires measuring function (HAQ) and quality of life (SF-12)

\begin{tabular}{llll}
\hline & & \multicolumn{2}{l}{$S F-12 \dagger$} \\
\cline { 3 - 4 } Condition & HAQ* & Physical & Psychological \\
\hline Rheumatoid arthritis & $1.75(1.42$ to 2.08$) \ddagger$ & $29.1(21.9$ to 36.2$) \ddagger$ & $42.8(36.4$ to 49.2$)$ \\
Low back pain & $1.27(1.08$ to 1.48$) \ddagger$ & $32.4(28.0$ to 36.8$) \ddagger$ & $43.0(38.8$ to 47.2$)$ \\
Knee osteoarthritis & $1.29(1.08$ to 1.49$) \ddagger$ & $31.7(27.3$ to 36.1$) \ddagger$ & $43.9(39.8$ to 48.0$)$ \\
Hand osteoarthritis & $1.24(1.05$ to 1.44$)$ & $35.5(31.1$ to 39.8$)$ & $43.3(39.2$ to 47.5$)$ \\
Fibromyalgia & $1.30(1.09$ to 1.51$) \ddagger$ & $33.9(29.3$ to 38.5$)$ & $39.0(34.6$ to 43.4$) \ddagger$ \\
None of the above & $1.23(1.02$ to 1.44$)$ & $35.3(30.8$ to 39.9$)$ & $44.8(40.5$ to 49.2$)$ \\
\hline
\end{tabular}

The results are expressed as estimated mean with $95 \%$ confidence intervals after adjustment for covariates in a multifactorial ANOVA model.

${ }^{\star}$ Range $0-3$ where $0=$ total capacity to perform activities of daily living and $3=$ complete inability to perform any of them. The model was adjusted for sex, age, social class, and presence of any non-rheumatic chronic disease.

†Range 0-100 from worst to best situation both in physical and psychosocial domains of quality of life. The model for the physical component was adjusted for sex, age, social class, educational level, and presence of any non-rheumatic chronic disease. The model for the mental component was adjusted for sex and the presence of any non-rheumatic chronic disease. $\neq \mathrm{p}<0.01$.

Table 5 Doctor visits for musculoeskeletal problems, consumption of NSAIDs, and disability compensations in Spain with relation to specific musculoskeletal diseases

\begin{tabular}{lllll}
\hline & \multicolumn{2}{l}{ In the past year } & \\
\cline { 2 - 3 } Condition & \multicolumn{2}{l}{ Consulted a physician for MES } & Consumed & $\begin{array}{l}\text { Receive disability } \\
\text { compensations }\end{array}$ \\
\cline { 2 - 4 } & Any number & $\geqslant 2$ physicians & NSAIDs $>1$ mo & \\
\hline RA & $72.7^{\star}$ & 27.3 & $63.6^{\star}$ & 9.1 \\
Low back pain & $61.2^{\star}$ & $25.8^{\star}$ & $40.9^{\star}$ & $8.0^{\star}$ \\
Knee OA & $66.4^{\star}$ & $26.8^{\star}$ & $45.7^{\star}$ & $5.4^{\star}$ \\
Hand OA & $58.8^{\star}$ & $22.8^{\star}$ & $38.2^{\star}$ & $2.2^{\star}$ \\
FM & $76.9^{\star}$ & $42.3^{\star}$ & $55.8^{\star}$ & $7.7^{\star}$ \\
None of the above & $25.3^{\star}$ & $8.1^{\star}$ & $14.3^{\star}$ & $1.7^{\star}$ \\
\hline
\end{tabular}

${ }^{\star} \mathrm{p}<0.01 ; \dagger \mathrm{p}<0.05$

MES = musculoskeletal symptoms; NSAIDs = non-steroidal anti-inflammatory drugs; RA = rheumatoid arthritis; $\mathrm{OA}=$ osteoarthritis; $\mathrm{FM}=$ fibromyalgia. with age up to 70 years, after which all but RA declined with age. Interviewer bias in the ascertainment of cases was discarded for all the target diseases by logistic regression.

To test the association between the specific rheumatic diseases and the HAQ and SF-12 scores, we first studied the bivariate effect of different variables on these scores. The mean HAQ score of the sample was 0.26 (SD 0.42) and showed a skewed distribution with most of the scores close to $0\left(P_{75}=0.1\right)$. The factors associated with worse HAQ scores in the bivariate analysis were female sex $(\mathrm{p}<0.001$, Mann-Whitney test), age $\left(r_{\mathrm{s}}=0.469 ; \mathrm{p}<0.001\right)$, educational level below elementary $(\mathrm{p}<0.001$, Kruskal-Wallis test and Bonferroni's correction), low social class ( $p<0.001$, Kruskal-Wallis test and Bonferroni's correction), and having a diagnosis of a chronic disease $(\mathrm{p}<0.001$, Mann-Whitney). After introducing these variables into an ANOVA model, low educational attainment lost statistical significance and was thus discarded from subsequent models. All the other significant factors were included in all models in which HAQ score was the dependent variable. Mean SF-12 scores were 50.18 (SD 9.28) (physical component) and 49.67 (9.92) (mental component). The physical component of the SF-12 was influenced by age, chronic comorbidity, and social class (all at a p level $<0.001$ ), and by female sex and low study level $(p>0.001$ but $p<0.05)$. All these factors were introduced as covariates in models testing the association with the SF-12 physical component. With regard to the mental component, no association was found with educational level, social class, or age, and the only significant associations appeared to be with sex and with a diagnosis of any chronic disease; these latter variables were eventually used in the ANOVA models.

Three ANOVA models were studied, one with each component of the SF-12 and another with the HAQ score as dependent variables. In each model the previously mentioned covariates were controlled for. The presence of any chronic comorbidity was exchanged by the presence of any non-rheumatic comorbidity to make the results more comprehensible. The results of the adjusted ANOVA models are shown in table 4. All the conditions except hand OA significantly impaired the functional ability in the following order of magnitude: RA, FM, symptomatic knee OA, and low back pain. With regard to quality of life, FM was the only condition independently associated with a low score in the mental component of SF-12. The diseases that affected the physical aspects of the quality of life in order of magnitude were: RA, symptomatic knee OA, and low back pain. 
In total, $33.3 \%$ of the subjects in the sample had visited a physician for musculoskeletal problems in the past year. Extrapolating to Spain's adult population, more than 10 million people consult a physician with a problem related to the musculoskeletal system annually. Subjects classified as having any of the target conditions consulted more frequently and consumed more NSAIDs than those without $(p<0.01$, table 5$)$. The proportion of people receiving disability compensations was significantly greater in those classified as having any of the target conditions than in those without (table 5). This association was maintained even after controlling for age older than 40 .

\section{Discussion}

The EPISER study was an initiative of the Spanish Society of Rheumatology to update figures on rheumatic diseases that had been obtained from small local studies. This is probably the first study with a national probabilistic sample addressed specifically to study the impact of rheumatic diseases in the general population in terms of prevalence, impact on function and work status, and on quality of life. This study enables a comparison to be made of the burden of rheumatic diseases with that of other common chronic conditions.

The sample selected was representative of the Spanish general population, despite only a moderate response rate. Data on the city register were not always accurate-in most the last mandatory update was carried out in 1996which resulted in a significant proportion of people having moved or died or unable to be located. This is particularly problematic in surveys such as the EPISER in which replacements were not used. The characteristics of those who could not be located were not very different from the rest of the sample. In fact, the rejection rate after successful contact was not high and the only difference found between those who declined and those who agreed to participate was the type of residence, probably a result of many local factors unrelated to having rheumatic disease.

As in other occidental populations, the prevalence estimates of FM and of low back pain were expectedly high. The prevalence of symptomatic knee OA $(10.2 \%)$ was considerably higher than the previously reported figure of approximately $6 \%,{ }^{20}$ which led us to analyse these results more closely (manuscript in preparation). The estimated prevalence of RA $(0.5 \%)$ was low compared with the rates of $1 \%$ in the USA and $0.8 \%$ in Central Europe. ${ }^{21}$ An even lower prevalence $(0.1 \%)$ was reported in a French study, although the approach was by mailed questionnaire and cases of inactive RA with actual deformities, as in our study, were not included. ${ }^{22}$ Evidence is accumulatingmostly in the form of communications to scientific meetings - of a more benign disease in Mediterranean countries which is also probably less prevalent.

Other surveys in Spain have shown a high prevalence of musculoskeletal pain and rheumatism in general, especially in the elderly, ${ }^{23}{ }^{24}$ but very few studies have addressed the epidemiology of specific rheumatic diseases. One such study conducted in the 1980s in a small town in the Spanish province of Ciudad Real examined 950 subjects over the age of 10 ( $86.6 \%$ of the eligible population in the village) and reported the prevalence of specific diseases such as OA $(26.1 \%)$, RA (1.6\%), and osteoporosis $(2.3 \%) .{ }^{25}$ Another study carried out in 1990 in Asturias, a Northern region of Spain, in which 702 subjects selected by random cluster sampling were interviewed and examined by rheumatologists, reported the prevalence of OA $(23.8 \%)$, low back pain (14.8\%), and "inflammatory disease" $(2.1 \%) .{ }^{26}$ More recently, two groups in the region of Navarra have investigated the prevalence of RA in the general population $(0.3 \%)$ and the prevalence and characteristics of FM in women $(1.5 \%),{ }^{27}{ }^{28}$ and a third group has studied the prevalence of RA in two rural towns in the province of Cordoba (0.3\%). ${ }^{29}$ Most of the results obtained in the EPISER study agree with these previous studies - that is, in Spain the prevalence of peripheral OA is high, the frequency of soft tissue rheumatism is comparable, and the prevalence of RA is low compared with other countries.

An interesting finding of the study was the poor agreement between having a rheumatic disease and perceiving oneself as rheumatic. Many of those who believed they had a rheumatic disease actually could not be classified under any of the more frequent musculoskeletal conditions. On the other hand, people never diagnosed as being rheumatic actually presented with clear signs and symptoms of one of the target diseases. This phenomenon may be related to the poor level of understanding of these diseases in the general population and their popular association with the normal aging process.

EPISER has confirmed the great impact of musculoskeletal diseases in the general population. Compared with persons without any of the target rheumatic diseases, and after controlling for other factors that may interfere with functional ability or with the health related quality of life, three diseases-RA, low back pain, and knee $\mathrm{OA}$ - were found to have a clearly detrimental effect on the lives of the affected subjects. In Spain musculoskeletal diseases are an important cause of work disability $^{30}$ and one third of the adult population of Spain visited at least one physician for musculoskeletal problems in the past year. This represents over 10 million visits in one year. Furthermore, among those classified as having any of the target diseases, many visited more than one doctor for their problem. Data from the Spanish National Health Institute show that during 1996 over 400000 hospital admissions (about 13.7\%) were related to musculoskeletal conditions. ${ }^{31}$ In addition, the total number of packs of NSAIDs and analgesics sold in Spain during the past year (over 80 million) significantly exceeded the packages sold for any other group of medical product. $^{32}$ 
The data in this study show that rheumatic diseases in Spain are an important source of pain, disability, and impaired quality of life to the individual, consume a large quantity of health resources (doctor visits, hospital admissions, medical product costs), and are a burden to society in terms of time off work. It is therefore unclear why rheumatic diseases do not demand more attention from public health authorities.

\section{Appendix: Members of the EPISER Study Group}

I Aretxabala, Hospital de Cruces, Bilbao; J Beltrán, Hospital General, Castellón; P Benito, Hospital del Mar, Barcelona; S Benito, C Hospital "San Millán-San Pedro", Logroño; M Calabozo, Hospital de Cruces, Barakaldo; J C Cobeta, Hospital "Obispo Polanco", Teruel; M Ciria, Hospital del Mar, Barcelona; C Fernández-Carballido, Hospital "Dr Peset”, Valencia; J A Fernández, Hospital Central de Asturias, Oviedo; J L Fernández-Sueiro, Hospital "Juan Canalejo", La Coruña; G Garrido, Hospital "Gregorio Marañón", Madrid; Y Grandal, Hospital General, Jerez de la Frontera; J Graña, Hospital "Juan Canalejo", La Coruña; A Hernández, Hospital "Juan Canalejo", La Coruña; C Hernández, Hospital C "San Carlos", Madrid; A Humbría, Hospital de la Princesa, Madrid; A Juan Mas, Hospital "Son Dureta", Mallorca; A Laiz, Hossspital "Santa Creu i Sant Pau", Barcelona; J López-Martínez, Social Psychology Department, Universidad Autónoma de Madrid, Madrid; O Martínez, Hospital de la "Santísima Trinidad", Salamanca; J Medina, Hospital General, Soria; M Menchón, Hospital "Virgen de la Arrixaca", Murcia; M Moreno, Hospital "Santa María del Rosell”, Murcia; T Navío, Madrid; F Navarro, Hospital "Virgen Macarena", Sevilla; A M Ortiz, Hospital de la Princesa, Madrid; B Ribas, Hospital "Sant Joan de Déu", Mallorca; P Rojas, Toledo; C Rodríguez-Lozano, Hospital "Doctor Negrin", Gran Canaria; F I Romero, Medical Advisor to Aventis Pharma; B Romero, Teruel; E Ruiz, Hospital de Cruces, Barakaldo; J M Salazar, Hospital "Infanta Cristina", Badajoz; J Sampedro, Hospital "Virgen de la Salud", Toledo; E Trujillo, Hospital General Universitario, Tenerife; N del Val, Hospital General, Soria; J P Valdazo, Hospital "Virgen de la Concha", Zamora; M Valverde, Preventive Medicine and Public Health Area, Universidad de Almería, Almería; J Vidal, Hospital General y Universitario, Guadalajara; V Villaverde, Hospital "La Paz", Madrid; E Yelin, Arthritis Research Group, University of California, San Francisco, USA.

EPISER was supported by a grant from the Fondo de Investigaciones de la Seguridad Social (FIS 99/0251) and sponsored by Merck Sharp and Dohme España. The authors appreciate the Merck Sharp and Dohme España. The authors appreciate the invaluable help from the personnel at the government of the cit-
ies selected in the study and at the health centres that provided the time and the space for the survey. The logistics of the survey the time and the space for the survey. The logistics of the survey Madrid, Spain.

1 Centers for Disease Control and Prevention. Arthritis prevalence and activity limitations. United States, 1990. MMWR 1994;43:433-8.

2 Reynolds D, Chambers L, Badley E, Bennet K, Goldsmith C, Jamieson E, et al. Physical disability among Canadians reporting musculoskeletal diseases. J Rheumatol 1992;19: $1020-1030$

3 Badley E, Tennant A. Impact of disablement due to rheumatic disorders in a British population: estimates of severity and prevalence from the calderdale rheumatic disablement survey. Ann Rheum Dis 1993;52:6-13.

4 Wang PP, Elsbett-Koeppen R, Geng G, Badley EM. Arthritis prevalence and place of birth: findings from the 1994 Canadian National Population Health Survey. Am J Epidemiol 2000;152:442-5.

5 Chaiamnuay P, Darmawan J, Muirden KD, Assawatanabodee P. Epidemiology of rheumatic disease in rural Thailand: a WHO-ILAR COPCORD study. J Rheumato 1998;25:1382-7.
6 Reves Llerena GA, Guibert Toledano M, González Otero $\mathrm{ZA}$, Alcocer Varela J, Cardiel MH. Prevalence of ZA, Alcocer Varela J, Cardiel MH. Prevalence of musculoskeletal complaints in Cuba. A community-based Rheumatol 2000;18:739-42.

7 Faroogi A, Gibson T. Prevalence of the major rheumatic disorders in the adult population of north Pakistan. Br J Rheumatol 1998;37:491-5.

8 Dunlop DD, Manheim LM, Song J, Chang RW. Arthritis prevalence and activity limitations in older adults. Arthritis Rheum 2001;44:212-21.

9 Batlle-Gualda E, Jovani Casano V, Ivorra Cortés J, Pascual Gómez E. [Musculoskeletal diseases in Spain. magnitude and specialised human resources.] Rev Esp Reumatol 1998;25:91-105.

10 Hill CL, Parsons J, Taylor A, Leach G. Health related quality of life in a population sample with arthritis. J Rheumatol 1999;26:2029-35.

11 Instituto Nacional de Estadística. España en cifras, 1999. www.ine.es/espcif/ espcifes/espcifes $99 . h$ htm

12 Alonso J, Pérez S, Saez M, Murillo C. [Validity of the occupation as social class indicator, according to the classification by the British Registrar General.] Gac Sanit 1997;11: $205-13$

13 Gandek B, Ware JE, Aaronson NK, Apolone G, Bjorner JB, Brazier JE, et al. Cross-validation of item selection and scoring for the SF-12 Health Survey in nine countries: results from the IQOLA project. J Clin Epidemiol 1998;51:1171-8.

14 Esteve-Vives J, Batlle-Gualda E, Reig A. Spanish version of the Health Assessment Questionnaire: reliability, validity, and transcultural equivalency. Grupo para la adaptación del HAQ a la población Española. J Rheumatol 1993;20: 2116-22.

15 MacGregor AJ, Riste LK, Hazes JMW, Silman AJ. Low prevalence of rheumatoid arthritis in Black-Caribbeans compared with Whites in inner city Manchester. Ann Rheum Dis 1994;53:293-7.

16 Altman R, Alarcon G, Appelrouth D, Bloch D, Borenstein $\mathrm{D}$, Brandt $\mathrm{K}$, et al. The American College of Rheumatology criteria for the classification and reporting of osteoarthritis of the hand. Arthritis Rheum 1990;33:1601-10.

17 Altman R, Asch E, Bloch D, Bole G, Borenstein D, Brandt $\mathrm{K}$, et al. Development of the criteria for the classification and reporting of osteoarthritis. Classification of osteoarthritis of the knee. Arthritis Rheum 1986;29:1039-49.

18 Wolfe $\mathrm{F}$ and the Multicenter Criteria Committee. The American College of Rheumatology 1990 criteria for the classification of fibromyalgia. Arthritis Rheum 1990;33: $160-72$.

19 MacGregor AJ, Ollier WPR, Silman AJ. Modification of ACR classification criteria for rheumatoid arthritis for use in population studies. Br J Rheumatol 1992;21(suppl): 37.

20 Felson DT, Naimark A, Anderson J, Kazis L, Castelli W, Meenan RF. The prevalence of knee osteoarthritis in the elderly. The Framingham Osteoarthritis study. Arthritis Rheum 1987;30:914-8.

21 Silman AJ, Horchberg MC. Epidemiology of the rheumatic diseases. Oxford: Oxford University Press, 1993.

22 Guillemin F, Briancon S, Klein JM, Sauleau E, Pourel J. Low incidence of rheumatoid arthritis in France. Scand $\mathbf{J}$ Rheumatol 1994;23:264-8.

23 Gabinete de Estudios Sociológicos Bernard Krief. [Reumatos 90: social and health study on rheumatic diseases in Spain]. Sociedad Española de Reumatología, Liga Reumatológica Española, Pfizer, Madrid, 1992.

24 Espallargués M, Alonso J, Ruigómez A, Antó JM. [Osteoarticular problems in the elderly: an approach to their population impact.] Med Clin (Barc) 1996;106:601-6.

25 Paulino J, Pinedo A, Wong C, Crespo D. [General study on the frequency of rheumatic diseases in a determined population with epidemiological aim.] Rev Esp Reumatol 1982; 9:1-8.

26 Ballina García FJ, Hernández Mejía R, Martín Lascuevas P, Fernández Santana J, Cueto Espinar A. Epidemiology of musculoskeletal complaints and use of health services in musculoskeletal complaints and use of health services

27 Ibáñez Bosch R, Garciarena Ezquerra LJ, Rodríguez Sanz de Galdeano M, Sandúa Sada M, Turumbay Ranz FJ, Castresana Arrate MV, et al. [Prevalence study of rheumatoid arthritis in the general population of Tudela's Health Area.] arthritis in the general population of

28 García del Río JA, Cortés Ugalde F, Agreda Peiró J, Sucunza Azcona A, Loaysa Lara JR, Tinturé Irigoyen T. [Prevalence of wide spread pain and of fibromyalgia among women.] Aten Primaria 2000;25(suppl):1.

29 Martínez Sánchez FG, González Domínguez J, Amian M, Puntas MD, Salmoral A, Escudero A, et al. [Prevalence study on rheumatoid arthritis in a rural area.] Rev Esp Reumatol 2000;5:179.

30 Tornero Molina J, Rodríguez Benito U, Massip M, Vidal Fuentes J, Fernández Echevarría. [Rheumatic disease as the cause of permanent work disability in Spain.] Rev Esp Reumatol 1992:19:294-7.

31 Ministerio de Sanidad y Consumo. CMBD/GDR-SNSProcedimientos. www.msc.es/cmbd/explotaciones/home.htm, 2000

32 Información Terapéutica del Sistema Nacional de Salud. [Therapeutic groups and active principles of greatest consumption in the National Health System during 1999. Informacion Terapéutica del Sistema 2000;24:73-6. 